\title{
Polysèmes
}

Revue d'études intertextuelles et intermédiales

\section{Quelques spectres et revenants dans un poème de Seamus Heaney}

\section{Cornelius Crowley}

\section{(2) OpenEdition}

\section{Journals}

Édition électronique

URL : http://journals.openedition.org/polysemes/1684

DOI : 10.4000/polysemes. 1684

ISSN : 2496-4212

Éditeur

SAIT

Édition imprimée

Date de publication : 1 janvier 2005

Pagination : 229-247

ISSN : 0999-4203

\section{Référence électronique}

Cornelius Crowley, "Quelques spectres et revenants dans un poème de Seamus Heaney », Polysèmes [En ligne], 7| 2005, mis en ligne le 01 janvier 2005, consulté le 04 mai 2019. URL : http:// journals.openedition.org/polysemes/1684; DOI : 10.4000/polysemes.1684

Ce document a été généré automatiquement le 4 mai 2019.

Polysèmes 


\title{
Quelques spectres et revenants dans un poème de Seamus Heaney
}

\author{
Cornelius Crowley
}

D'abord ce poème de Seamus Heaney, puis une histoire de bus :

Two Lorries

It's raining on black coal and warm wet ashes.

There are tyre-marks in the yard, Agnew's old lorry

Has its cribs down and Agnew the coalman

With his Belfast accent's sweet-talking my mother.

Would she ever go to a film in Magherafelt?

But it's raining and he still has half the load

To deliver farther on. This time the lode

Our coal came from was silk-black, so the ashes

Will be the silkiest white. The Magherafelt

(Via Toomebridge) bus goes by. The half-stripped lorry

With its emptied, folded coal-bags moves my mother:

The tasty ways of a leather-aproned coalman!

And films no less! The conceit of a coalman...

She goes back in and gets out the black lead

And emery paper, this nineteen-forties mother,

All business round her stove, half-wiping ashes

With a backhand from her cheek as the bolted lorry

Gets revved and turned and heads for Magherafelt

And the last delivery. Oh, Magherafelt!

oh, dream of red plush and a city coalman

As time fastforwards and a different lorry

Groans into shot, up Broad Street, with a payload

That will blow the bus station to dust and ashes...

After that happened, I'd a vision of my mother,

A revenant on the bench where I would meet her

In that cold-floored waiting-room in Magherafelt,

Her shopping bags full up with shovelled ashes.

Death walked out past her like a dust-faced coalman 
Refolding body-bags, plying his load

Empty upon empty, in a flurry

of motes and engine-revs, but which lorry

Was it now? Young Agnew's or that other;

Heavier, deadlier one, set to explode

In a time beyond her time in Magherafelt...

So tally bags and sweet-talk darkness, coalman.

Listen to the rain spit in new ashes

As you heft a load of dust that was Magherafelt,

Then reappear from your lorry as my mother's

Dreamboat coalman filmed in silk-white ashes. (13-14)

A man and a woman are waiting for a bus at a request stop. He remains silent, but she keeps talking to him, or rather at him, for she accuses him, in no uncertain terms, of having made a pass at her. Her insults, her threats, her attempts at striking an alliance with other members of the queue founder upon his inscrutable silence. The more he remains quiet, the more she talks, exposing her frailty, her need for affection, her anxiety, and anger.

When the bus eventually comes, he boards it, and she remains by the stop, and immediately attempts to seduce the first man who joins the queue. (Lecercle 224) ${ }^{1}$

2 L'idiome le plus banal - mais parfaitement adéquat - dont nous disposons pour parler de la métaphore comporte deux termes : teneur et véhicule. Si on fait de la métaphore la mère de toutes les figures, et du langage poétique un emploi se caractérisant par le recours à celle-ci, nous nous trouvons d'emblée embarqués dans une réflexion sur le poème où nous l'envisageons comme étant, par son essence ou son télos, un mode de transport. Lu à la suite du poème de Heaney, ce micro-récit cité par Jean-Jacques Lecercle ${ }^{2}$ délivre une teneur inquiétante, surgie du croisement avec le poème, mais qui était déjà là, n'attendant qu'à naître, latente dans cette histoire d'autobus, un peu trop symétrique pour être une triviale tranche de vie. On remarquera l'homme resté sans parole, alors que la femme s'épuise à parler, ne parvenant ainsi qu'à exhiber sa faiblesse, l'impuissance d'une parole à conjurer cette présence muette qui l'indispose. Et puis il y a ce retournement des rôles : ayant refusé de monter à bord, elle essaiera à son tour de jouer de sa propre force séductrice vis-à-vis du premier homme venu.

3 Nombreux sont les contes et tableaux qui figurent la mort sous la guise d'une belle dame qui croise un cavalier, non pas pour le dévier de son droit chemin, plutôt pour le précipiter vers l'ultime station, depuis toujours prévue d'avance. Comme si c'était le chemin si bien balisé de l'entreprise intentionnelle - exercice de la rationalité instrumentale en vue de la transformation du monde vécu: et peu importe que l'entreprise soit celle d'un chevalier en quête du Graal ou d'un commis voyageur en quête de clients - soudain s'avoue comme un détournement provisoire, une tentative de différer l'ultime terme de ce droit chemin. Ce dispositif archétypal du conte - un chemin, une rencontre - nous est rappelé, avec toutefois une inversion des rôles attribués aux sexes, dans cette histoire d'un inconnu qui importune : belle dame, homme qui ne parle pas.

4 La métaphore de toutes les métaphores, c'est cette articulation entre une charge portée et un creux porteur ${ }^{3}$, en vue de l'envoi d'une charge vers un destinataire ou destination. Soit, dans le micro-récit, l'ordre silencieusement intimé de monter à bord, pour aller on ne sait où, selon le bon plaisir de Dieu sait quelle instance. Ce récit du transport au statut d'archi-métaphore semble attester la vanité de nos actes de langage : paroles proférées sur fond d'une communication rompue, exposition à une instance qu'aucune parole ne saurait apaiser, vaine parade. Nous transposons et déplaçons des mots - convoqués, 
disposés, envoyés -, à défaut de pouvoir parer ou porter les morts. Une lettre les sépare dans la langue française. "The end of art is peace » $(58)^{4}$, a pu écrire Heaney, laissant ainsi entendre que l'acte poétique qui consiste à façonner des figures porteuses d'une charge parfois « lourde à porter », comme on dit, aurait la vertu d'amadouer ou de charmer une instance autrement restée intraitable, pour faire passer un poids autrement intolérable. Là résiderait la gloire et la raison d'être du poème: faire passer ce que le langage ordinaire ne saurait supporter; apaiser des courroux ou des ressentiments restés à vif. Et le poème y parviendrait, en vertu de cette articulation que lui seul réalise, entre une charge à transporter et le véhicule poétiquement façonné, en vue de l'effet visé: un convoyage réussi. Car s'il reste dans ce monde tant d'agitation violente - si nous ne sommes pas en paix, et d'abord avec nous-mêmes -, ce serait par une accumulation de ressentiments, dont le poids ferait ployer tout acte de langage tenté selon les règles ordinaires de la communication " entre hommes et femmes de bonne volonté ", tout ça se traduisant par l'échec des négociations, à cause d'une accumulation ou d'un trop plein des blessures de l'histoire, que seule la parole poétique, mise en branle par ce silence énigmatique et par l'impuissance de la parole ordinaire, pourrait, à coup de ruses langagières - tropes en tout genre - à son tour déranger, en rétablissant des relations : une aimable conversation au lieu de la vaine apostrophe d'un autre qui se tait. «The end of art is peace ». C'est, me semble-t-il, un étonnant programme poétique. La poésie serait le seul art capable d'être à la fois une conversion - de la boue en or, chez le jeune Rimbaud, de la mauvaise conscience du survivant en célébration, chez Heaney - et conversation : institution d'un ordre humain où le symbolique pourrait enfin permettre la relève de la violence, par la pleine assomption de sa charge négative : «the nightmare of history » de Stephen Dedalus.

5 Soit l'hypothèse (pessimiste, sophiste) d'un maintien, sans solution de continuité, de la violence à travers le jeu d'une violence symbolique, la parole n'étant que la continuation de la violence en un autre registre. Soit, à l'opposé, l'hypothèse irénique d'une violence transcendée, au fur et à mesure de la pleine appropriation par les hommes d'une parole purgée de toute ruse sophistique, se conformant enfin à l'idée de la raison pratique : Soyez raisonnables, mettez-vous autour d'une table en femmes et hommes de bonne volonté. Soit, troisième hypothèse, l'hypothèse de la parole poétique envisagée comme la seule parole à même de faire passer certaines charges autrement trop lourdes ; la poésie, en vertu de sa stratégie figurale et cryptante, étant capable de conduire à bon port des secrets et douleurs que l'abord trop net ferait exploser ou revivre. "The end of art of peace ». Oui, mais une paix gagnée par un art suprême de la ruse, seul en mesure de vaincre l'art de la violence. Alors que la droiture d'un discours transparent de sincérité n'y parviendrait pas: la précipitation de la violence, coup pour coup, ayant sa réplique en une précipitation ou droiture trop nette de la parole voulant trop simplement dire le vrai des choses. L'apaisement passerait par la langue fourchue du poème. Belle hypothèse. Il y a une quatrième hypothèse, je la dirai à la fin.

6 Le micro-récit de l'arrêt de bus est introduit au dernier chapitre de The Violence of Language. Il y aurait, selon Jean-Jacques Lecercle, de la violence concomitante à tout acte de langage, dans la mesure où les relations à autrui sont rarement de l'ordre de la conversation amène : il y a des charges lourdes à porter ; celles-ci sont travesties, encore alourdies, du simple fait d'être transportés, mal reçues, renvoyées. La langue-véhicule aurait ses caches et ses faux planchers, par lesquels transiteraient des marchandises dont le locuteur ne se doute pas forcément. S'il subsiste, selon Jean-Jacques Lecercle, le reste, « 
the remainder " (6), c'est qu'il resterait toujours une teneur pas, ou mal, véhiculée: quelque chose ne passe pas, ou passe mal. Mais cela s'expliquerait pour des raisons concomitantes à la condition langagière: au réel du langage et de la condition symbolique. Ces conditions qui font qu'il y aura toujours un reste : le véhicule exige son dû, la charge doit d'abord être correctement conditionnée. La loi de ce conditionnement se sous-entend dans les vers de Keats : « Beauty is truth, truth beauty - that is all / Ye know on earth and all ye need to know $\|^{5}$. C'est-à-dire que si le poème doit se faire la forme cave où seront déposés les restes d'un être mort, comme dans une Grecian urn, il faudra que la forme fasse bonne figure: c'est sous le leurre d'une surface figurant - mais en même temps figeant - l'instant d'un amour pour toujours non-consommé que se conservent et se déposent les cendres du mort. L'art consommé du semblant figural, ce sera de faire superficiellement bonne figure, par déposition ${ }^{6}$ à la fois sa sauvegarde, mais aussi son éviction et son remplacement - de la teneur inconvenante : un art de la composition. Comme on peut composer avec son ennemi, parfois habilement, parfois en se pliant à la dictature de ses léonines conditions que l'on ferait mieux, peut-être, de rejeter au départ, en refusant de s'embarquer dans l'entreprise de la figuration. On peut estimer que si l'inactivation de la charge du négatif passe par le primat du véhicule sur sa teneur, à laquelle sont dictées les conditions de son acheminement figural, cette inactivation réussit trop bien à la fin. Trop de ruse figurale (autrement ça ne passe pas); trop de réussite, y compris dans la poésie de Seamus Heaney, à mesure que la teneur que l'on veut porter subit la loi de la conversion symbolique, alchimie du verbe. Je n'imagine pas que Jean-Jacques Lecercle souscrive à la troisième hypothèse, hypothèse d'une parole poétique qui, par ruse et par détours, aurait la force performative d'ouvrir à la relève de la violence. Mais je ne sais pas comment il se situe par rapport à la quatrième.

7 L'œuvre de Heaney, depuis le début, nous propose des apologues où le poète figure son propre art poétique, assumé comme un art heureux. Si on a pu s'interroger sur le «bonheur » de Mallarmé ${ }^{7}$, nul doute n'est possible concernant le bonheur esthétique de Heaney, qui cherche et qui trouve - façonne - des figures poétiques aussi domestiquement familières qu'elles sont appropriées. Les possibilités de cet art sont à la mesure de sa nécessité. À la différence de la cueillette des mûres qui, mises sans adjuvant dans un seau, ne connaissent que la conversion du pourrissement :

We hoarded the fresh berries in the byre.

But when the bath was filled we found a fur,

A rat-grey fungus, glutting on our cache.

The juice was stinking too. Once off the bush

The fruit fermented, the sweet flesh would turn sour. $(5)^{8}$

le poème éponyme, "Blackberry-Picking ", peut rendre présent l'avènement estival des mûres, conservant de l'événement sa primitive maturité. Les modèles de l'efficace poétique sont à portée de main :

As you plaited the harvest bow

You implicated the mellowed silence in you

In wheat that does not rust

But brightens as it tightens twist by twist

Into a knowable corona

A throwaway love-knot of straw (58) ${ }^{9}$

ou ils sont visibles dans le monde alentour, par exemple dans la maestria de l'oiseau en vol :

But when goldfinch or kingfisher rent

The veil of the usual,

Pinions whispered and braced 
As I stooped, unwieldy

And brimming,

my spurs at the ready. (199) ${ }^{10}$

Depuis le début, Heaney aura mesuré ce qu'il peut faire de ce don, arpentant son domaine poétique où, de sa ferme installation en un lieu et en un moment présent, il sait célébrer des ancêtres ou creuser dans un sol, avec la même certitude de toujours trouver qu'un sourcier cherchant de l'eau. Notons le décrochage, aussi léger qu'absolu, dans le poème « Digging » (1966):

Under my window, a clean rasping sound When the spade sinks into gravelly ground:

My father, digging. I look down

Till his straining rump among the flowerbeds

Bends low, comes up twenty years away

Stooping in rhythm through potato drills

Where he was digging. (I) ${ }^{11}$

De même, depuis le début, Heaney aura sondé les possibilités et les limites de l'empathie poétique: "Docker " (Death of a Naturalist, 1966) dessine la caricature d'un ouvrier protestant des chantiers navals de Belfast; "A Constable Calls» (North, 1975) décrit un franchissement de seuil depuis « l'autre côté » religieux, un franchissement qui n'est pas pour autant un accueil. Et toujours, avec leurs divers motifs, mais presque toujours domestiques, et d'un ordinaire désarmant, il y a ces poèmes qui dressent la constitution de l'art poétique de Heaney: sous quelles conditions sera franchi le seuil ? Quels titres faire valoir en vue d'un permis de séjour dans la maison de la poésie ${ }^{12}$ ? Une autre contribution à cet art poétique évoquera l'art de bâtir (fait de modestie et de robustesse) du père ${ }^{13}$. Être, c'est habiter, selon Heidegger : la figuration poétique dresse des formes où les êtres chers trouvent encore refuge après la perte de leur enveloppe de chair. Heaney continue à figurer la possibilité d'une survie - une vie après la vie - à travers l'articulation traditionnelle entre soma et sema, d'un noyau de sens préservé intact à travers les épreuves. Et toujours ces poèmes disent deux choses qui, mises en boucle, ne font qu'une, par leur tressage d'une forme qui préserve et contient, en même temps qu'elle exclut et met à distance. D'abord il y a ces deux questions : question de la violence et question de la figuration, la dernière étant d'abord entendue selon l'hypothèse où la violence serait l'épreuve-limite pour tout acte de symbolisation; puis, très vite, le pressentiment qu'il n'y a qu'une question, celle d'une inextricable collusion entre la violence et la figuration poétique, à travers le faire symbolique qui vise et qui réussit à faire sens, en faisant bon accueil dans le poème à ceux qui nous sont proches. C'est à peu près la quatrième hypothèse, évoquée plus haut: en amont de toute réflexion sur la forme poétique, sur les possibilités et les limites esthétiques, pointer une configuration humaine qui fut tracée à coups de séparations et de lignes de frontière - entre l'animal et l'homme, entre une souche autochtone et les autres. Configuration forcément duelle, où il $\mathrm{y}$ aura toujours teneur et véhicule, semblant et profondeur, comme il y aura deux camions et deux convois dans le poème de Heaney : grotte ou chaumière pour les vivants hôtes, tombeau pour les morts, un foyer de sens d'autant plus résistant qu'il en impose à tout pénétrant. Et c'est pourquoi nous lirons les mots suivants, qui viennent clore un livre d'Ernst Fischer :

Man, who became man through work, who stepped out of the animal kingdom as transformer of the natural into the artificial, who became the magician, man the creator of social reality: not until humanity itself dies will art die ${ }^{14}$ 
En ajoutant hélas ceci : «not until humanity itself dies will violence end. », les deux allant de pair, figuration symbolique des humains et violence des humains, tout autant que beauté et vérité, dans la mesure où l'acte poétique, jeu d'ajustement entre teneur et véhicule, dit la quintessence de toute entreprise symbolique, qui vise l'établissement de soi comme un être chez soi en un (son) lieu, à travers l'élaboration de ces formes, tant matérielles que symboliques, permettant d'ajuster le degré d'exposition au monde, exposition qui, trop large, aboutira à l'extinction du sujet candide. Nous ne dirons jamais de Heaney qu'il est candide. Il sait d'instinct doser l'aperture de la porte et le franchissement du seuil, en veillant à ce que la maison de la poésie tienne bon, car le sens de l'accueil passe par la maitrise des hôtes. Si on veut discerner ce qui reste de la violence, une fois résolue ou éliminée toute violence dont la détermination serait économique ou objectivement sociale, si on veut en toucher la quintessence, savoir ce qu'il en reste après le raffinement d'une analyse sociale bien menée, comme les cendres blanches comme soie restent dans l'âtre autour duquel officie la mère du poète, nous dirons que ce qui reste, c'est la difficile confrontation à retardement entre l'entreprise d'installation et de mise en forme et tout ce qui n'y aura trouvé accueil : le reste, l'exclu, le refoulé, tout ce dont on ne veut ou ne peut pas tenir compte, lorsque la fin visée est la mise en ordre réussie d'un domaine politique ou d'un domaine poétique. La figuration poétique appelle d'autres poèmes, écrits contre des poèmes existants; les entreprises d'appropriation et d'établissement laissent des ressentiments, préludes à d'autres actes d'expropriation. Dans la rationalité économique du capitalisme, on voudrait restreindre ce jeu instable à la logique régissant la production et l'échange des choses : destruction créatrice, selon Schumpeter. Dans les enjeux plus primitifs d'installation dans un lieu (c'est l'enjeu tristement primitif des " troubles » en Ulster, dont il vaut mieux, selon Heaney, ne rien dire, "Whatever you Say, Say Nothing $»^{15}$ ), cette instabilité pressentie est source de méfiance dans l'accueil et le franchissement de seuil : tant dans la maison habitée que dans le poème qui abrite ces vivants et ces morts qui sont les siens. Où le pouvoir poétique d'accueillir et de réanimer les restes dépend de la vigilance dans l'admission - des motifs, des mots, des morts -, préalable à l'œuvre de conversion figurale : car si Heaney est un grand poète, on ne dira pas de lui qu'il prend à bras-le-corps le défi qui consisterait à penser poétiquement le capitalisme mondialisé. Il n'est pas vrai par contre que la violence ne saurait trouver son arrangement figurai. Car le poème est un foyer (hearth) où trouver abri, sous condition d'une admissibilité selon les règles. Or ces règles sont une application dans le discours des règles découlant des liens de sang. L'ensemble de ces conditions - possibilité et nécessité $\mathrm{du}$ poème, le don de déplacement temporel que celui-ci possède, les limites de l'hospitalité poétique - sous-tendent la formidable assurance qui est audible dans les vers suivants, célébration d'une filiation réussie :

1.1 .87

Dangerous pavements.

But I face the ice this year

With my father's stick. $(20)^{16}$

\section{«Two Lorries »}

11 Deux camions donc, à quarante ans d'intervalle, dans ce nouvel apologue de l'entreprise poétique, le lien entre les deux véhicules s'effectuant avec autant de maîtrise que dans « Digging », où l'intervalle est moindre, où la relation s'établit entre deux avatars du père 
encore vivant. Dans ce poème publié en 1996, les parents sont morts, et la maturité et la force poétiques de Heaney passent par la capacité de veiller sur l'ombre de la mère, comme elle a autrefois veillé sur lui. Renversement des rôles mais, comme toujours chez Heaney, sans rupture de la transmission. Heaney marche droit avec la canne du père; il peut également se faire une sorte de déesse tutélaire, car il sait agencer des corps poétiques dont le mode d'accueil s'apparente au corps maternel : comme la mère lui avait fait place avant qu'il ne vienne à la parole, ses formes poétiques, les tombeaux qu'il façonne, sont désormais là pour accueillir l'ombre d'un revenant, pour que la mère, maintenant morte, ne soit point condamnée à une errance sans feu ni lieu. On notera comment ce poème est construit comme une succession d'opérations de convoyage : un camion apporte son chargement de charbon destiné au foyer, lieu-omphalos de la maisonnée où veille la mère, garante de la paisible installation du jeune garçon, déjà établi en son rôle de veilleur, étant l'observateur de cette rencontre avec Agnew, avant d'être à son tour le gardien des ombres et revenants. Il y a les sacs, pleins ou vides, de charbon ou de restes déchiquetés, car la modernité technologique de la guerre a fait importer en Ulster les body-bags, ces travestissements du travail de consignation et de conservation que veut réaliser le poète. Et n'oublions pas ce lieu de la tentation refusée, noire cavité du cinéma, la salle obscure : «dream of red plush », lieu dit en des mots dont la douceur voluptueusement chuintée entre en résonance avec le toponyme langoureusement répété dans le poème, Magherafelt, promesse d'un possible toucher feeling et douceur de feutrine-, envisagé, mais sitôt éloigné, refusé. Il n'empêche : le poème se permet un moment d'étourdissement où le pouvoir poétique de Heaney risque de chuter, chose qui n'arrivera pas, tant qu'il garde la canne de son père et qu'il reste debout, n'envisageant pas la mère autrement que comme "this nineteen-forties mother ", qui ne pourra que plaisanter à propos d'un camionneur de Belfast à la parole si douce. (Agnew, dont l'invitation sera, bien sûr, déclinée, porte un nom catholique qui assure son admission dans la maison de la poésie). L'assurance propre au pouvoir poétique de Heaney, et qui n'aura jamais connu de défaillance, est de l'ordre de la certitude domestique : une assurance ayant pour contrepartie une certitude concernant ceux qui ont été là pour veiller sur lui, avant que la veille poétique sur les morts ne succède à la pieuse veille de la mère, qui l'aura rendu possible.

Un camion fait surgir un autre camion, à quarante ans d'intervalle. En fait c'est le camion dont la charge fait exploser une ville, du coup convertie en reste - « a load of dust that was Magherafelt »- qui, par traumatisme et à retardement, fera revenir le camion des années quarante. Ce détour par un autre temps et un autre camion est le seul, mais efficace, trope permettant de faire poétiquement accueil à un attentat commis dans le présent de cette fin d'enfance (lorsque les parents ne sont plus là pour veiller). Si la fin de l'art est la paix, l'apaisement peut venir dans ce poème, mais à la condition que Heaney sache jouer un camion de l'enfance et de la sécurité domestique contre le camion de la terreur aléatoire. Tant que la matrice symbolique fonctionne encore, Heaney peut transfigurer tout événement, fût-ce de la mort par terreur. Tant que la matrice fonctionne, le poète peut convoquer et transfigurer Agnew, le charbonnier d'antan, pour en faire le bienveillant batelier qui aidera ces morts venus importuner le poète dans leur passage à l'autre rive.

Or cet acte poétique consommé, s'il suscitera toujours l'admiration du lecteur, laisse aussi un malaise, le sentiment qu'il existe peut-être une grâce poétique permettant de s'installer fermement en son domaine, mais également une déshérence antipoétique, une terre vaste de l'imaginaire figural : d'un côté le pouvoir poétique de tout traiter et de tout 
transfigurer, un don se traduisant par le maintien par le poète de son propre équilibre, alors qu'il accueille les débris de ce monde dans les tombeaux qu'il aura poétiquement façonnés; d'un autre côté une affliction qui rend impossible d'accorder pleinement crédit à ce pouvoir de transfigurer douleurs et blessures en faisant accueil à ces restes en quête de paix. Toujours est-il que Heaney ne sera pas le poète d'une aphasie; la bouche ne restera pas bée devant la charge à porter et la chose à dire.

Il n'y a aucune raison de douter que la forme poétique, exprès creusée pour accueillir et pour porter des charges, comme le camion ou le bus, ou comme le corps maternel, ne puisse prendre en elle les phénomènes d'un monde, celui de Heaney, dont le centre est Mossbawn, la ferme où il naît; où tout ce qui advient - y compris la désolation de la violence terroriste - est susceptible d'accueil, du moment qu'il y a résonance, qu'une corde est touchée dans l'imaginaire matriciel du poète.

Et c'est là qu'il y a peut-être problème : quel poème faire avec l'image d'un pistolet posé à la tempe d'un rebelle africain, dont le corps mort va s'écrouler? Que faire lorsqu'il n'est nullement nécessaire de passer par un déplacement temporel, «as time fastforwards ", car l'événement de la mort surgira dans le même plan, l'instant après dans la même séquence? La question est naïve, elle suppose une demande faite à la poésie de remplir une fonction que ni la politique ni la religion ne sauraient remplir. Et pour cause : poésie, politique, religion, participent de la même entreprise symbolique, inductrice de violences perpétrées contre les autres intrus, alors même qu'elles permettent l'anamorphose figurale qui peut rendre invisible - en tant que violence - la violence infligée à ces autres intrus, tout en auréolant l'ombre de ceux parmi les siens qui ont péri. Alors que l'arrêt sur l'image de ce mort ne peut que nous sidérer - c'est donc si peu de chose ? - et que cette mort filmée, dont la vision est reproductible par manipulation d'une machine, travestit le mode de figuration des amants dans le poème de Keats, car dans ce cas présent la visibilité interminablement reproductible stoppe toute tentative d'accueil en soi, comme si se vérifiait encore et toujours, face au trop visible, ce que pressentait le sujet poétique wordsworthien, lorsqu'il s'expose à Bartholomew's Fair dans The Prelude ${ }^{17}$. Mais si, chez Heaney, la figuration poétique - retraitement de tout phénomène, jusqu'au plus intraitable, jusqu'à la mort par la terreur, selon un strict protocole d'accueil - possède des pouvoirs certains, que la résonance familiale reste puissante, on verra là le signe de la grandeur de Heaney, mais on y lira aussi le pressentiment que la violence est consubstantielle à une condition humaine, à laquelle est également consubstantielle une logique symbolique procédant par rattachement de tout phénomène nouvellement advenu à la matrice, plus ou moins prégnante, déjà là dans l'espace social d'une culture, ou dans l'espace imaginaire du poète, qui ne peut que relever d'une culture. (Heaney ne semble nourrir ni utopie universaliste ni fausse pudeur à ce sujet : il revendique le foyer qui lui assure son «slow papish burn ») $)^{18}$.

16 La poésie serait un art défensif. Il en serait de même de toute élaboration symbolique: acte défensif et conservateur, d'autant plus puissant dans ses stratégies d'accueil qu'il saura établir ses lignes de démarcation, sans possibilité d'infiltration, et sans que soit brouillée la confrontation nette entre le proche - accueilli - et l'étranger, tenu à distance. La maîtrise démontrée dans la figuration d'un domaine proche où, d'intime résonance en intime résonance, tout peut s'apprivoiser, aurait pour corrélat la possibilité de concevoir l'acte poétique comme une fidélité maintenue à l'égard de ses propres morts, et l'impossibilité concomitante de concevoir une poétique se pratiquant contre les règles de l'inscription affective. 

question de savoir si la violence met en déroute l'élaboration figurale, dont la poésie est l'un des modes d'exercice, on répondra que non. C'est, au contraire, en opposant ses facultés de remembrement au démembrement de la violence que la poésie s'autorise une audience et une raison d'être communales. Mais si la question est de savoir si la poésie, charme et splendeur figurale, affranchirait d'une logique de violence consubstantielle à l'histoire humaine, il est tout aussi évident que non. Car la reconnaissance d'une dette vis-à-vis d'une instance étrangère qui ne nous affecterait pas intimement - se mettre, par exemple, à figurer poétiquement les ombres de ceux qui meurent par la violence, mais qui ne sont pas, comme on dit, « des nôtres » - ferait baisser l'éclat poétique, conduirait à une déperdition de la chaleur de ce foyer de sens. Au point que la poésie ne subsisterait plus comme réussite figurale, et qu'il ne resterait qu'un soupir de lassitude. Voilà ce qui ne serait plus de l'ordre de la figuration, n'étant que l'aveu d'une impuissance.

Car la quatrième hypothèse rejoint - mais superficiellement - la première hypothèse (cynique) : celle d'une continuité entre une violence gestuelle et une violence symbolique, dont le poème, en tant que discours, pourrait être une expression active, avec des effets qui diffèrent selon les lieux de sa réception. L'hypothèse que j'avance ici ne suppose pas un sujet intentionnel, collectif ou individuel, en mesure de jouer à sa guise de ces deux leviers: tantôt de la violence matérielle, tantôt de la violence symbolique. J'essaie d'envisager, en la prenant au sérieux, mais sans y voir la moindre raison de célébrer notre humanité, l'hypothèse formulée par Ernst Fischer: tant que l'humanité existe, l'art existera en tant qu'entreprise symbolique. Mais en y ajoutant un corollaire concernant le statut liminaire de la violence: à la fois reste laissé en dehors de cette enceinte symbolique où nous aimons nous reconnaître et nous " ressourcer »; mais aussi, et tout autant, une force consubstantielle au surinvestissement symbolique, dont la magie sympathique peut nous laisser indemnes de tout sentiment, lorsque la violence perpétrée s'effectue en violation de la chair de ceux qui, en termes d'univers symbolique inventé ou de notre espace familier habitable, ne seraient pas « des nôtres ».

19 Je garde plusieurs souvenirs du début des événements en Irlande du nord. D'un professeur lisant ces lignes du $4 \mathrm{e}$ acte de Macbeth: «I think our country sinks beneath the yoke. It weeps, it bleeds, and each new day a gash/Is added to her wounds ", nous proposant la pièce de Shakespeare comme un véhicule (détourné) capable de transmettre la teneur de ce qui arrivait à «notre pays ». Autre souvenir, celui d'une pièce " expérimentale», une création collective appelée, "Violence as a mode of transport ", où il me semble maintenant qu'à travers la charge manifeste - la question de la violence politique - passait en contrebande une teneur secrète, le récit d'amours interdites entre des garçons adolescents. Je rappellerai aussi que le conflit en Ulster a donné lieu à une curieuse invention linguistique : le terme proxy bomb, une bombe par procuration ou par vicariance. Un véhicule est détourné de sa première destination, puis chargé d'une teneur explosive, potentiellement mortelle, qu'un innocent conducteur devait conduire à sa destination, en le faisant passer à travers le cordon des barrages militaires, sous la menace indirecte faite aux membres de sa propre famille. Une nouvelle fois, nous retrouvons notre articulation entre teneur et véhicule.

20 Nous avons voulu jouer notre rôle de destinataire possible d'un poème de Heaney, en chargeant notre lecture de quelques réflexions générales sur le lien indéfectible entre violence et figuration. En la chargeant, aussi, de quelques sentiments et souvenirs plus particuliers : sentiment de gâchis dont on peut être habité maintenant, en aval de ces 
événements appelés "The Troubles»; souvenir d'avoir entendu un étudiant américain dire, vers 1972, " the trouble with you Irish people is that you don't know how to compromise", et de la jubilation que l'on pouvait cultiver à ne pas se situer dans l'espace du compromis ; souvenir de l'interrogation, récurrente dans les journaux du dimanche à partir de 1969, environ : " where are the poets, since we're living in this time of violence? ». Car vivant sur un héritage yeatsien, il paraissait évident que toute violence rendait à la fois possible et nécessaire sa conversion symbolique et sa relève.

L'articulation entre nécessité et possibilité du poème recoupe l'articulation entre violence et figure : la première rend nécessaire la seconde, qui, par bonheur, s'avère possible, dans ce meilleur des mondes symboliques. Mais si telle est l'indépassable règle symbolique, nous dirons que toute conversion en figure fait obliquement référence à sa part de violence, non pas comme à son reste non-traité, mais comme à la condition de son inévitable avoir-lieu comme figuration, toujours un peu trop bien réussi.

\section{NOTES}

1. Jean-Jacques Lecercle, The Violence of Language, Londres, Routledge, 1990.

2. Voir Lecercle, 224 et $263 \mathrm{n}$. Le passage cité est la présentation par Jean-Jacques Lecercle de «Request Stop », une pièce courte de Harold Pinter, publiée dans A Slight Ache and Other Plays, London, Methuen, 1961.

3. Je cite, de mémoire, un terme employé par Roger Chazal lors d'une réunion du groupe « Tropismes ", Paris X, Nanterre.

4. Le vers figure en italiques dans le poème « The Harvest Bow », Field Work, London, Faber, 1979.

5. Derniers vers de « Ode on a Grecian Urn » de Keats.

6. Louis Marin, « Déposition du temps dans la représentation peinte », De la représentation, Paris, Gallimard/Éditions du Seuils, coll. « Hautes Études », 1994.

7. Gérard Genette, « Bonheur de Mallarmé ? », Figures I, Paris, Éditions du Seuil, 1966, 91.

8. « Blackberry-Picking » (1966), New Selected Poems 1966-1987, London, Faber, 1990.

9. «The Harvest Bow », op. cit.

10. «Drifting Off », (1984) New Selected Poems, 1987.

11. Selected Poems, 1.

12. «From the Frontier of Writing ", The Haw Lantern, London, 1987, 6.

13. «Crossings » xxxiii, Seeing Things, London, Faber, 1991, 91.

14. J'utilise l'édition anglaise, trad. Anna Bostock, Ernst, Fischer, The Necessity of Art: A Marxist Approach, Londres, Pelican, 225.

15. Titre d'un poème souvent cité, North, 1975.

16. Seeing Things.

17. Wordsworth, ThePrelude, Book VII, 1, 650-740.

18. "Poetry is bom out of the watermarks and colourings of the self. But that self in some ways takes its spiritual pulse from the inward spiritual structure of the community to which it belongs; and the community to which I belong is Catholic and nationalist; I believe that the poet's force now, and hopefully in the future, is to maintain the efficacy of his own "mythos", his own cultural and political colourings, rather than to serve any particular momentary strategy that his political leaders, his paramilitary organisation or his own liberal self might want him to serve. I 
think that poetry and politics are, in different ways, an articulation, an ordering, a giving of form to inchoate pieties, prejudices, world-view, or whatever. And I think that my own poetry is a kind of slow, obstinate, papish bum, emanating from the ground I was brought up on ", Seamus Heaney, « Dialogue with Seamus Deane », The Crane Bag, 1, Dublin, 1977, 67.

INDEX

oeuvrecitee Two Lorries 